\title{
On the Distribution of the Number of Vertices of a Random Polygon
}

\author{
By
}

\section{Christian Buchta}

(Vorgelegt in der Sitzung der math.-nat. Klasse am 11. Dezember 2003 durch das w. M. August Florian)

\begin{abstract}
Assume that $n$ points $P_{1}, \ldots, P_{n}$ are distributed independently and uniformly in the triangle with vertices $(0,1),(0,0)$, and $(1,0)$. Consider the convex hull of $(0,1)$, $P_{1}, \ldots, P_{n}$, and $(1,0)$. Denote by $N_{n}$ the number of those points $P_{1}, \ldots, P_{n}$ which are vertices. Let $p_{k}^{(n)}(k=1, \ldots, n)$ be the probability that $N_{n}=k$. BÁRÁNY, Rote, SteigeR, and ZHANG [1] proved that $p_{n}^{(n)}=2^{n} /[n !(n+1) !]$. We derive for $k=1, \ldots, n-1$ the values of $p_{k}^{(n)}$ and thus obtain the exact distribution of $N_{n}$. Knowing this distribution provides the key to the answer of some long-standing questions in geometrical probability, e.g., to the distribution of the number of vertices of the convex hull of $n$ points distributed independently and uniformly in a convex polygon.
\end{abstract}

Mathematics Subject Classification (2000): 52A22, 60D05.

Key words: Convex hull, random points, random polygons.

$*$

Theorem 1. For $n \in \mathbb{N}$ and $k=1, \ldots, n$ the probabilities $p_{k}^{(n)}$ are given by

$$
p_{k}^{(n)}=\frac{2}{n(n+1)} \sum_{j=k-1}^{n-1}(n-j) p_{k-1}^{(j)}
$$


with $p_{0}^{(0)}=1$ and $p_{0}^{(j)}=0$ for $j \in \mathbb{N}$. Alternatively, these probabilities are given by

$p_{k}^{(n)}=2^{k} \sum \frac{i_{1} \cdots i_{k}}{i_{1}\left(i_{1}+1\right)\left(i_{1}+i_{2}\right)\left(i_{1}+i_{2}+1\right) \cdots\left(i_{1}+\cdots+i_{k}\right)\left(i_{1}+\cdots+i_{k}+1\right)}$,

where the sum is taken over all $i_{1}, \ldots, i_{k} \in \mathbb{N}$ such that $i_{1}+\cdots+i_{k}=$ $n$.

The proof of Theorem 1 will be published in a forthcoming paper, which will also describe how the distribution of the number of vertices of the convex hull of $n$ points distributed independently and uniformly in a convex polygon arises in terms of the probabilities $p_{k}^{(n)}$. Here we will only state the following consequence of Theorem 1:

Theorem 2. The expected value and the variance of the random variable $N_{n}$ are given by

$$
\begin{aligned}
E N_{n} & =\frac{1}{3}\left(2 \sum_{k=1}^{n} \frac{1}{k}+1\right), \\
\operatorname{var} N_{n} & =\frac{1}{27}\left(10 \sum_{k=1}^{n} \frac{1}{k}+12 \sum_{k=1}^{n} \frac{1}{k^{2}}-28+\frac{12}{n+1}\right) .
\end{aligned}
$$

The asymptotic version of the first formula in Theorem 2, i.e. $E N_{n} \sim \frac{2}{3} \log n$ as $n$ tends to infinity, is a classical result due to RÉNYI and SULANKE [4]. The asymptotic version of the second formula, i.e. $\operatorname{var} N_{n} \sim \frac{10}{27} \log n$ as $n$ tends to infinity, is due to GROENEBOOM [3]. It was obtained by approximating the process of vertices of the convex hull of a uniform sample by the process of extreme points of a realization of a Poisson point process: The "left-lower boundary" of the convex hull of a uniform sample of size $n$ from the interior of the square $[0, \sqrt{n}]^{2}$ is associated with the "left-lower boundary" of the convex hull of a realization of a Poisson point process on $\mathbb{R}_{+}^{2}$ with intensity Lebesgue measure. For comments on GROENEBOOM's paper see [2].

\section{References}

[1] BárÁny, I., Rote, G., Steiger, W., Zhang, C.-H. (2000) A central limit theorem for convex chains in the square. Discrete Comput. Geom. 23: 35-50

[2] BuchtA, C. (to appear) An identity relating moments of functionals of convex hulls. Discrete Comput. Geom. 
[3] Groeneboom, P. (1988) Limit theorems for convex hulls. Probab. Theory Relat. Fields 79: $327-368$

[4] RÉNYI, A., SULANKE, R. (1963) Über die konvexe Hülle von $n$ zufällig gewählten Punkten. Z. Wahrscheinlichkeitsth. Verw. Geb. 2: 75-84

Author's address: Prof. Dr. Christian Buchta, Institut für Mathematik, Universität Salzburg, Hellbrunner Straße 34, 5020 Salzburg, Austria. 